\title{
Localization and Synthesis of $\alpha$-Fetoprotein in the Chicken
}

\author{
B. Slade and J. Milne* \\ Department of Zoology, University College of Wales, Aberystwyth, Dyfed, U.K.
}

\begin{abstract}
Summary. The localization and sites of synthesis of $\alpha$-fetoprotein in chick embryos throughout development have been investigated using the combined techniques of immunofluorescence microscopy and tissue culture in the presence of radiolabelled amino acids, followed by immunoautoradiographic analysis.

Alpha-fetoprotein is present in a range of embryonic tissues and especially concentrated in the yolk sac, liver and connective tissue. Analysis of culture fluids revealed that the yolk sac is the major site of $\alpha$-fetoprotein synthesis with smaller, but significant quantities being produced by the liver.

These results are discussed in relation to mammalian $\alpha$-fetoprotein, and the merits of the chick embryo for studies on the biological function of AFP are considered.
\end{abstract}

Key words: Alpha-fetoprotein - Chicken - Immunoautoradiography Immunofluorescence microscopy.

\section{Introduction}

Embryo-specific $\alpha$-fetoproteins (AFP's) have been detected in the sera of all mammals so far investigated (Tartarinov and Afanasyeva, 1965; Gitlin and Boesman, 1967 a, b) and more recently in the shark (Gitlin, 1974). Serum proteins specific for the embryonic phase have also been described in the chicken (Weller, 1966; Gitlin and Kitzes, 1967), and the most abundant of these is considered to be the avian homologue of mammalian AFP (Lindgren et al., 1975). Not only does the serum concentration and the ontogenetic pattern of chicken AFP resemble that of mammals, but it also has a similar molecular weight and amino acid composition (Ruoslahti and Pihko, 1975).

Send offprint requests to: Dr. B. Slade, Department of Zoology, University College of Wales, Aberystwyth, SY 23 3DA, Dyfed, U.K.

* Supported by an award from the Science Research Council, to whom grateful acknowledgement is made 
The synthesis of AFP in mammals, as determined by immunoautoradiographic analysis of tissue culture fluids, is confined to the embryonic yolk sac and liver, with an additional small contribution in the human from the gastrointestinal tract (Gitlin et al., 1972). Similar studies in the shark have revealed that the liver and stomach are the major sites of synthesis, with variable quantities also being produced by the yolk sac, intestine and kidney (Gitlin, 1974). However, in the chicken Gitlin and Kitzes (1967) concluded that only the yolk sac was actively involved in AFP synthesis. Although the liver was active in protein synthesis they failed to detect AFP production by this organ. Recently, Weller (1976) has demonstrated AFP synthesis by the liver using a similar technique to that employed in the present study.

Immunofluorescent studies in various species have tended to implicate these same tissues in AFP synthesis, but additional high concentrations are also found in a wide range of connective tissues clearly not involved in synthesis (Linder and Seppala, 1968; Linder et al., 1971; Branch and Wild, 1972).

This study has attempted to correlate the sites of AFP localisation in the developing chick embryo, as revealed by immunofluorescent staining, with results indicative of active synthesis derived from in vitro culture of a range of embryonic tissues in the presence of radiolabelled amino acids followed by immunoautoradiographic analysis.

\section{Materials and Methods}

Preparation of Antisera

Antiserum to embryonic chick serum (a-ECS) was prepared in rabbits by subcutaneous injection of $0.5 \mathrm{ml}$ pooled serum in complete Freund's adjuvant. Two booster injections in incomplete adjuvant were given at fortnightly intervals. Hyperimmune animals were bled 10 days after the last injection and a $\gamma$-globulin enriched fraction prepared from the resultant antiserum by ammonium sulphate precipitation.

Antiserum specific for chick AFP (a-AFP) was prepared according to a similar schedule using an AFP enriched serum fraction derived from Sephadex G 200 chromatography of pooled embryonic chick serum, followed by absorption with Sepharose-linked concanavalin-A (Pagé, 1973). The resulting antiserum was made monospecific by sequential absorption against insolubilised adult chicken serum and egg white from infertile eggs according to the method of Avrameas and Ternynck (1969) (Fig. 1).

Fluorescein labelled sheep anti-rabbit $\gamma$-globulin (A-RGG-FITC) was obtained commercially (Wellcome).

\section{Fluorescence Microscopy}

Whole 5 day chick embryos and samples of liver, intestine, kidney, heart, brain and yolk sac from older embryos were fixed in cold $95 \%$ ethanol and processed for fluorescent microscopy according to the method of Sainte-Marie (1962). Frozen sections of some tissues were also prepared, fixed in cold $100 \%$ acetone and air dried. All sections were equilibrated in phosphate buffered saline (PBS) and treated with $1 \%$ sodium nitrite in $2 \%$ acetic acid to reduce non-specific antibody binding (Burtin and Sabine, 1972) prior to incubation with a-AFP. After a thorough washing in PBS the sections were treated with a-RGG-FITC, washed, mounted in buffered glycerol and viewed under a Zeiss U.V. microscope. Control sections were treated with a-RGG-FITC only. Photographic records were made on Kodak Panatomic-X film.

\section{Immunoautoradiographic Analysis}

Embryos were removed from eggs at various stages of incubation under sterile conditions. Tissues and organs required for culture were dissected into Eagles basal medium. After mincing into approxi- 


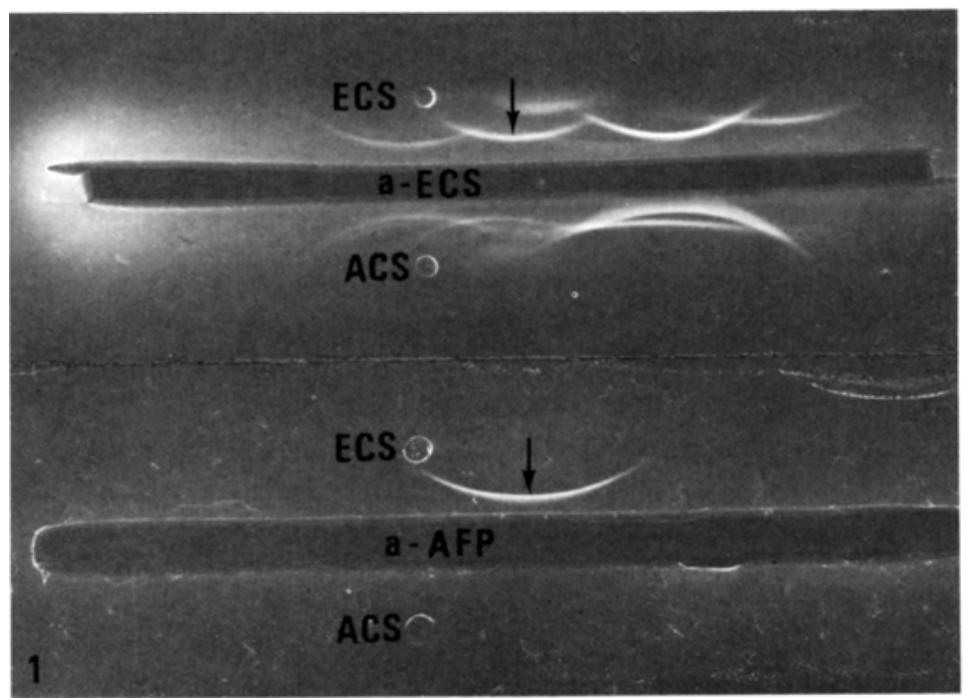

Fig. 1. Immunoelectrophoretogram of adult chick serum $(A C S)$ and embryonic chick serum $(E C S)$ developed with rabbit antisera to embryonic chick serum (a-ECS) and chick AFP (a-AFP). Arrow: AFP

mately $1 \mathrm{~mm}^{3}$ pieces, between 50 and $100 \mathrm{mg}$ of tissue were placed into universal bottles containing $2 \mathrm{ml}$ bicarborate buffered Eagles plus $5 \%$ fetal calf serum and $2 \mu \mathrm{Ci}$ (U-14 C) protein hydrolysate (Radiochemical Centre, Amersham). Cultures were gassed with $5 \% \mathrm{CO}_{2}$ in air and incubated for 4 days with continuous rolling at $37^{\circ} \mathrm{C}$. Control cultures included adult tissues, embryonic tissues killed by the addition of $0.1 \%$ sodium azide, and carrier embryonic serum alone. After incubation the cultures were frozen and thawed twice and centrifuged at $9,000 \mathrm{G}$ to remove cellular debris. The clear supernatants were dialysed against running tap water for $48 \mathrm{~h}$ and freeze dried.

Samples were reconstituted in $50 \mu \mathrm{l}$ of pooled 14-16 day embryonic chick serum immediately before immunoelectrophoresis, which was performed on microscope slides after the method described by Scheidegger (1955). Precipitin arcs were developed with rabbit antiserum to embryonic chick serum. The slides were exhaustively washed in several changes of saline and finally dried overnight in an incubator at $37^{\circ} \mathrm{C}$. The dried slides were placed in contact with Kodak X-ray film and allowed to expose at $4^{\circ} \mathrm{C}$ for 14 days. The contact-autoradiographs thus obtained were developed in Kodak D 19 developer and the immunoelectrophoresis slides were stained with amido black.

\section{Results}

\section{Immunofluorescent Localization of AFP}

Sections of embryonic membranes from 5, 10,15 and 20 day embryos consistently displayed specific fluorescence indicative of AFP (Fig. 2). In the yolk sac prominent fluorescent vesicles were present in the endodermal cells, but since they were also to be seen in control sections (Fig. 3) they were identified at autofluorescent yolk droplets. AFP was diffusely distributed throughout the endodermal cells, the underlying basement membrane and the vascular mesenchyme. The vascular endothelium was intensely stained and aggregated fluorescent material was also seen in the blood vessels. The chorion and amnion showed similar diffuse full- 

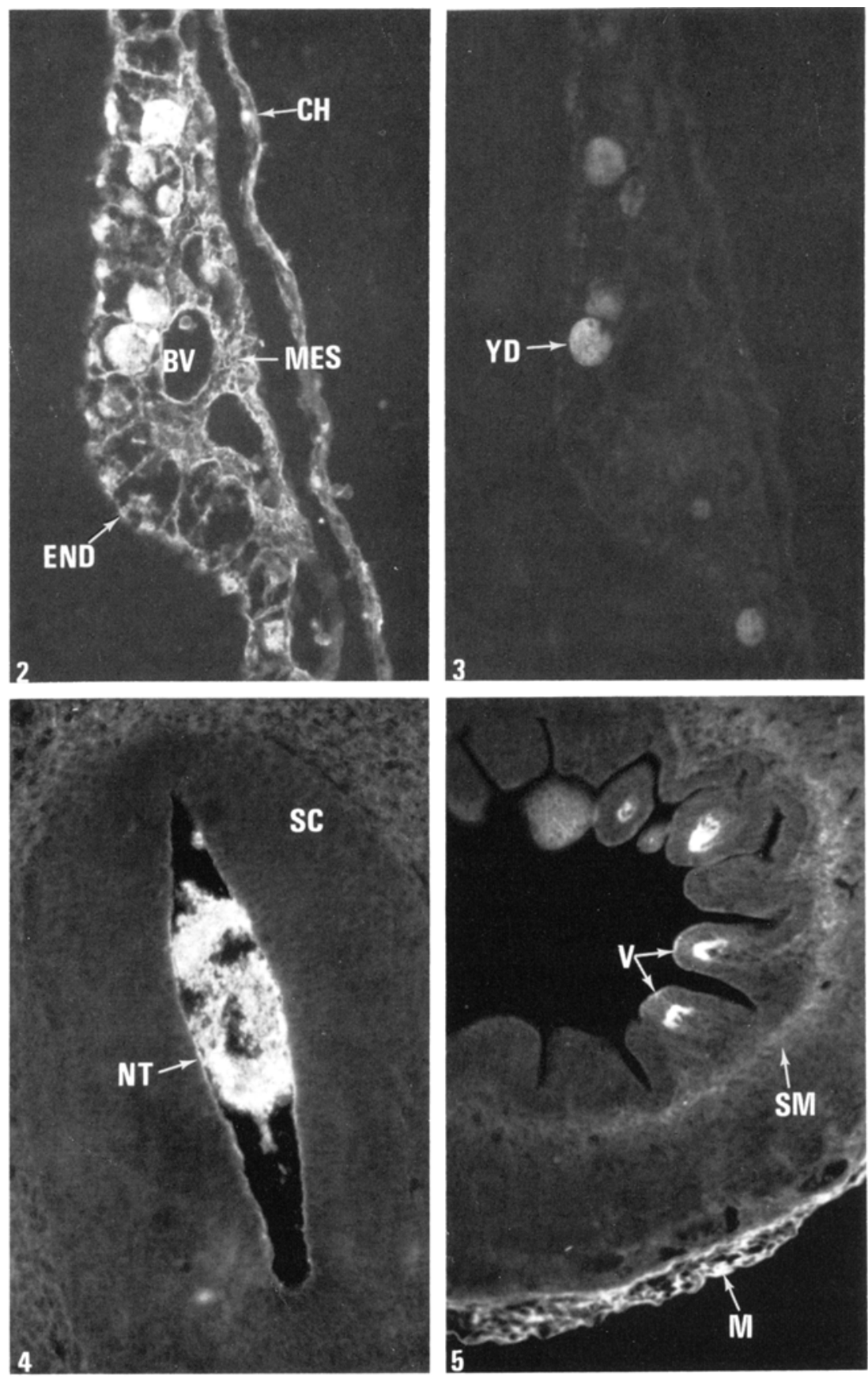

Fig. 2. Immunofluorescent localization of AFP within the yolk sac. Diffuse staining throughout the endoderm $(E N D)$ and vascular mesenchyme $(M E S)$ is evident. The chorion $(C H)$ also stains intensely. Blood vessel $(B V) . \times 90$

Fig. 3. Control yolk sac shows autofluorescent yolk droplets $(Y D)$ within endodermal cells. $\times 90$

Fig. 4. Section through the neural tube (NT) of a $5 \mathrm{D}$ embryo shows intense staining of the contents of the neural canal. Faint fluorescence can also be seen in the adjacent sclerotome $(S C) . \times 90$

Fig. 5. Section through $15 \mathrm{D}$ intestine showing intense staining of some villi $(V)$. The submucosa $(S M)$ and intestinal mesentery $(M)$ also stain intensely. $\times 90$ 


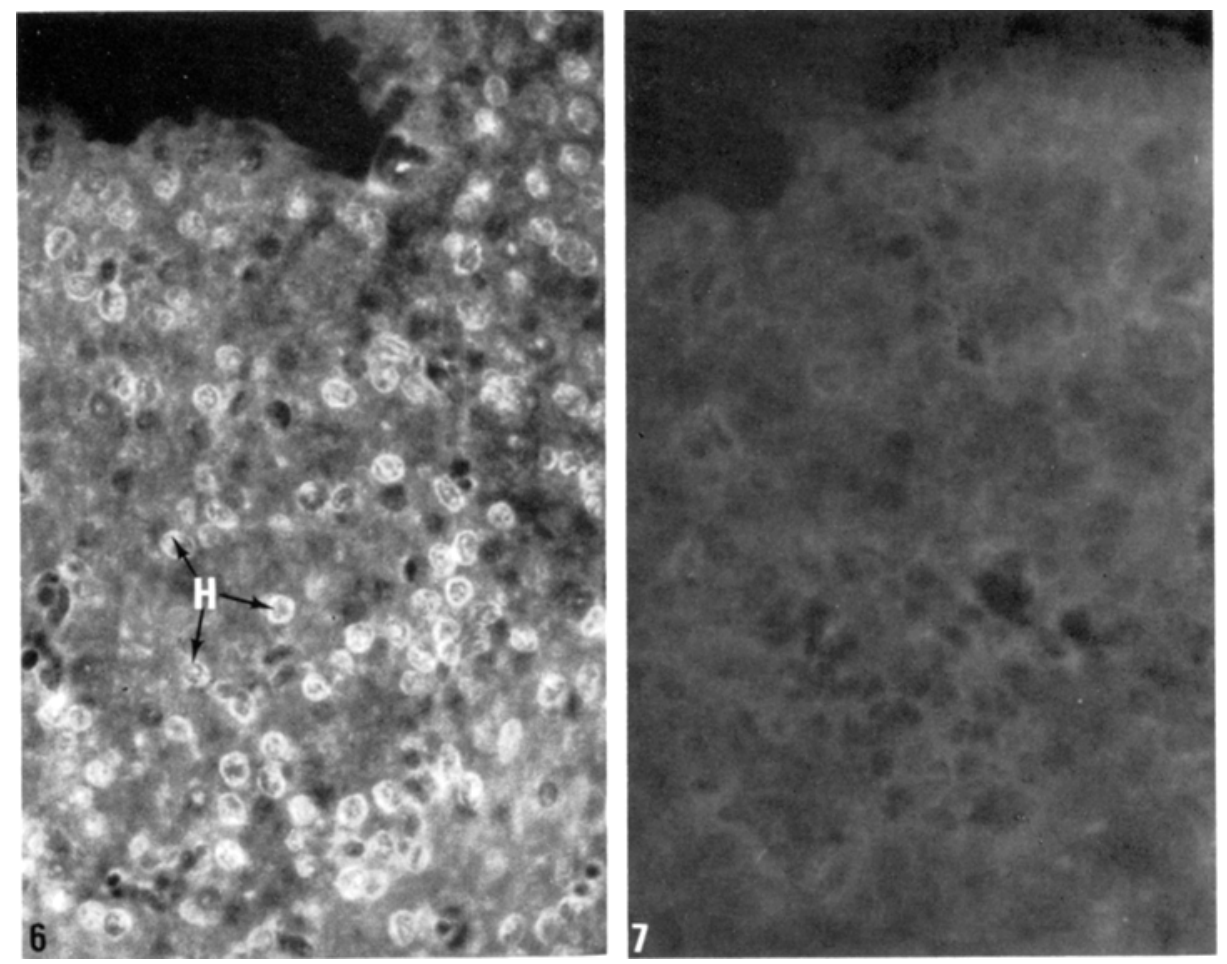

Fig. 6. Immunofluorescent localization of AFP within a frozen section of $15 \mathrm{D}$ liver. The majority of hepatocytes $(H)$ show fluorescent staining indicative of AFP. $\times 250$

Fig. 7. Control liver section showing absence of hepatocyte staining. $\times 250$

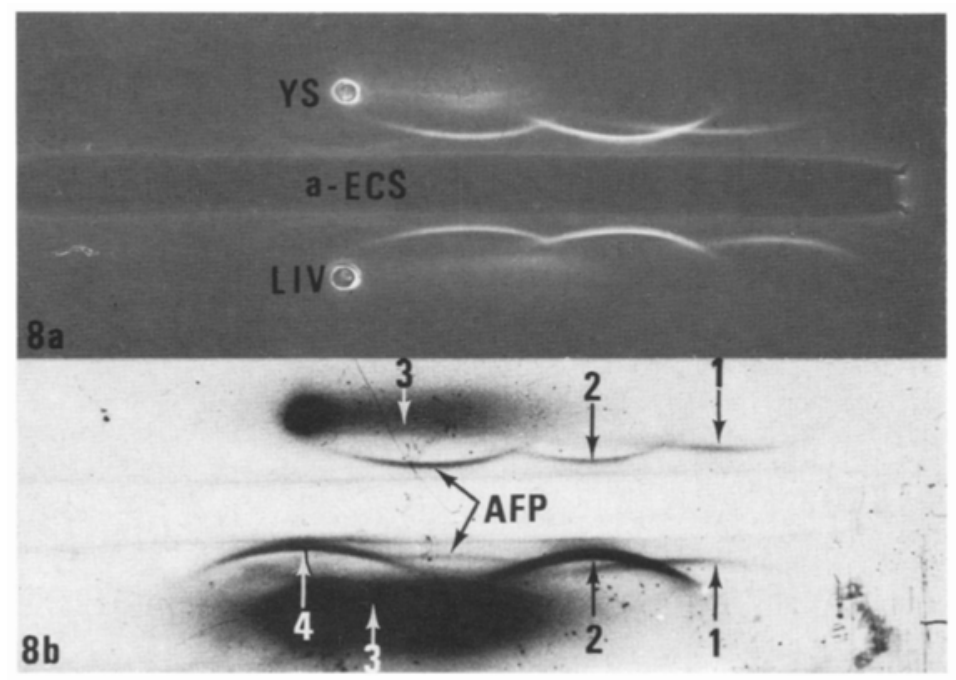

Fig. 8. a Immunoelectrophoretogram of tissue extracts from cultures of 15D yolk sac (YS) and liver ( $L I V$ ) with added carrier embryonic serum developed with rabbit anti-embryonic chick serum (a-ECS). b Contact radioimmunoelectrophoretogram of above slide. Note the marked incorporation of labelled precursors into $A F P$ in the yolk sac extract. The liver evidently synthesises a wider range of proteins and relatively less AFP. 1 Prealbumin; 2 Albumin; 3 Chylomicron region; 4 Conalbumin 
width staining, with occasional deposits of adherent fluorescent material derived from the exocoelomic fluid.

Sections of whole 5-day embryos revealed specific fluorescent staining throughout the mesenchyme and connective tissue. The ventricles of the brain and the neural canal showed intense fluorescence (Fig. 4) indicating that the fluids within these cavities were rich in AFP. The nervous tissue itself fluoresced only weakly. The contents of the optic capsule and also the corneal mesoderm fluoresced brightly but the nervous tissue of the retina did not. The densely packed sclerotome tissue surrounding the notochord and neural tube displayed varying degrees of fluorescence.

Small amounts of specific fluorescence were associated with the intestines of 10,15 and 20-day embryos. This varied from weak staining of the submucosa at 10 days, to patchy staining of the intestinal villi at later stages (Fig. 5).

Paraffin sections of liver revealed no specific fluorescence, but frozen sections showed localization of AFP in the majority of hepatocytes and adherent to the surface of erythrocytes within the lumen of blood vessels (Figs. 6, 7). The only fluorescence seen in sections of kidney and heart was that associated with connective tissue and blood vessels.

In vitro Synthesis of $A F P$

Immunoelectrophoretic autoradiographic analysis of the culture fluids of all the tissues examined revealed that AFP was produced only by the yolk sac, embryonic liver and possibly the embryonic intestines.

Judged by the intensity of the autoradiographic images the yolk sac was the major site of AFP synthesis (Fig. 8). Other proteins, especially albumin, prealbumin and conalbumin were also produced. Cultures of 5 and 10-day embryonic membranes produced mainly AFP, only traces of other proteins being detected. At 15 days AFP was still produced in significant quantities, but relatively more albumin and prealbumin could also be detected. Cultures of 20-day membranes produced no AFP but variable quantities of other proteins were present. All cultures producedan intense diffuse autoradiographic image in the chylomicron region.

Cultures of minced 5 day embryos produced traces of prealbumin and conalbumin but no AFP. A variety of proteins were synthesised by cultures of liver from 10,15 and 20-day embryos (Fig. 8). The most abundant proteins were albumin, prealbumin and conalbumin, with smaller quantities of other proteins including AFP, $\beta$-lipoprotein and an unidentified $\alpha_{2}$ globulin also present.

With the possible exception of intestine, none of the other tissues examined synthesised AFP, although all produced trace amounts of other proteins. In some 15 day intestine cultures a faint AFP band could be identified.

Control cultures containing embryonic serum alone showed very slight binding of labelled amino acids to albumin and AFP. Azide treated cultures produced no autoradiographic images.

\section{Discussion}

An inherent problem in the method whereby proteins are localized within tissue sections with fluorescent antibody, is that no distinction can be made between 
actual sites of synthesis and deposits of a secondary nature. This is particularly so in the case of serum proteins which are widely disseminated throughout the vascularized tissues of the body. However, if tissues are cultured in vitro under appropriate conditions, in the presence of radiolabelled amino acids, analysis of the tissue extracts and culture fluids will reveal any newly synthesised protein. These two methods have previously been successfully used together to identify the tissues involved in the synthesis of AFP and its sites of localization in rats (Gitlin and Boesman, 1967), rabbits (Branch and Wild, 1972) and humans (Gitlin et al., 1972). In this study a similar approach has been employed in the chick where an homologous embryo-specific protein has recently been described (Lindgren et al., 1975).

Results obtained by fluorescent microscopy consistently revealed AFP within the yolk sac at all stages of development studied. The distribution was diffuse within the endodermal cells, the basement membrane and vascular mesenchyme, very similar to the distribution described by Gitlin (1967) in the rat yolk sac, and in marked contrast to the distinct vesicular localization found in the rabbit yolk sac endoderm (Branch and Wild, 1972; Slade and Budd, 1972). The vascular endothelium was intensely stained and aggregated fluorescent material was also frequently seen in the blood vessels. Prominent fluorescent vesicles were present in the yolk sac endoderm, but since these were also present in control sections they were considered to be autofluorescent yolk particles. AFP was present in the yolk, as evidenced by double diffusion, and using immunofluorescent staining alone it was not possible to distinguish between the AFP synthesised by the yolk sac and that derived from the yolk by endocytosis. Branch and Wild (1972) drew attention to this problem in the rabbit where AFP was present in the uterine fluid, and its distribution in the yolk sac was indistinguishable from that of endocytosed protein derived from the uterine lumen.

A number of proteins were synthesised by the yolk sac in tissue culture including AFP, pre-albumin, albumin, conalbumin and occasionally a small amount of an unidentified $\alpha_{2}$-globulin. This range is essentially similar to that found in an earlier study by Gitlin and Kitzes (1967). Relatively large amounts of AFP were synthesised by 5,10 and 15-day cultures, but 20-day cultures failed to synthesise any. However, immunofluorescence revealed dense concentrations in 20-day yolk sac, suggesting that AFP persists in the tissue after cessation of synthesis.

A distinct ontogenetic pattern of AFP synthesis emerged from the results. As judged by the intensity of the autoradiographic images, AFP synthesis increased until 15D and then fell rapidly until at $20 \mathrm{D}$ none could be detected. This impression is supported by quantitative data on AFP levels in developing chick sera (unpublished observations) and is also consistent with the immunoelectrophoretic observations of Weller (1976). The rapid decline in serum AFP concentration after approximately $15 \mathrm{D}$ is partially accounted for by the appreciable increase in blood volume of the embryo at this time although it is also evident that synthesis by the yolk sac is decreasing.

Difficulty was experienced in localizing AFP in paraffin-embedded sections of liver. Similar problems have been reported by other workers (Engelhardt et al., 1971) and it is not clear why AFP-antigenic activity is poorly preserved in this tissue following ethanol fixation. Successful localization was achieved, however, 
using frozen sections fixed in cold acetone. Fluorescent staining indicative of AFP was confined to hepatocytes, the majority of which stained, in contrast to the sparse and somewhat scattered distribution of AFP-containing cells found in mammalian liver (Engelhardt et al., 1971; Branch and Wild, 1972). Autoradiographic analysis of culture media revealed that chick liver synthesised small amounts of AFP throughout development. In their earlier study Gitlin and Kitzes (1967) had failed to detect AFP synthesis by this organ. The relatively low level of synthesis indicated was surprising in view of the intensity of fluorescent staining and suggests that AFP is retained within hepatocytes for sometime after synthesis, as is evidently the case in yolk sac immediately before hatching.

The proportion of AFP synthesised by the chick liver in comparison with the yolk sac is somewhat less than in mammals. Gitlin (1974) suggested that a shift in sites of AFP synthesis has taken place during evolution. In the shark, the most primitive vertebrate in which AFP synthesis has been studied (Gitlin et al., 1973), the stomach and liver are the major sites of synthesis, smaller quantities being produced by the intestine and yolk sac. In mammals the liver is the predominant source, with the yolk sac making an important contribution in some species and the gastrointestinal tract producing very little. In birds the role of the liver in AFP synthesis is subordinated to the yolk sac and very little is produced by the intestine.

In common with mammals, AFP is the major serum protein during embryonic development in the chicken, suggesting an important, but as yet unknown physiological role. A number of properties have been ascribed to mammalian AFP, including oestrogen-binding capacity (Uriel et al., 1972), which has led to speculation that the protein may be involved in the hormonal control of development (Swartz and Soloff, 1974). Chick AFP does not bind oestrogen (unpublished observations), and since this property is by no means universal among mammals, it is unlikely to be of fundamental physiological significance. Recent experiments have demonstrated that mammalian AFP has immunosuppressive properties in vitro (Murgita and Tomasi, 1975a, b) and it has also been shown to bind to T lymphocytes (Dattwyler et al., 1975). These observations have prompted speculation that AFP might be important in preventing the fetus from immunological attack. However, the presence of an homologous protein in the chicken, no less important physiologically as judged by its abundance, would seem to cast serious doubt on this interpretation.

The chick embryo offers a valuable experimental system for investigations on the biological function of AFP. It has several advantages over the mammalian systems which have previously been used, including accessibility, accurate determination of developmental stage and isolation from maternal influences. We are currently investigating the effect of eliminating AFP with specific antibodies at different stages of development in an attempt to find out more about the role of AFP during embryogenesis.

\section{References}

Avrameas, S., Ternynck, T.: The cross-linking of proteins with glutaraldehyde and its use for the preparation of immunoadsorbents. Immunochemistry 6, 53-56 (1969) 
Branch, W.R., Wild, A.E.: Localisation and synthesis of $\alpha$-fetoprotein in the rabbit. Z. Zellforsch. 135, $501-516$ (1972)

Burtin, P., Sabine, M.C.: Inhibition of the non-specific fixation of fluorescent globulins. Rev. Europ. Etud. Clin. Biol. 17, 76-77 (1972)

Dattwyler, R.J., Murgita, R.A., Tomasi, T.B., Jr.: Binding of $\alpha$-fetoprotein to murine T cells. Nature (Lond.) 256, 656-657 (1975)

Engelhardt, N.V., Goussev, A.I., Shipova, L. Ja., Abelev, G.I.: Immunofluorescent study of alphafetoprotein ( $\alpha \mathrm{fp}$ ) in liver and liver tumour. I. Technique of $\alpha \mathrm{fp}$ localisation in tissue sections. Int. J. Cancer 7, 198-206 (1971)

Gitlin, D.: Phylogeny and ontogeny in the evolution of $\alpha$-fetoprotein compared to the emergence of the immunoglobulins. In: Masseyeff, L'Alpha-Feto-Proteine. Paris: Inserm 1974

Gitlin, D., Boesman, M.: Fetus-specific serum proteins in several mammals and their relation to human $\alpha$-fetoprotein. Comp. Biochem. Physiol. 21, 327-336 (1967a)

Gitlin, D., Boesman, M.: Sites of serum $\alpha$-fetoprotein synthesis in the human and rat. J. Clin. Invest. 46, 1010-1016 (1967 b)

Gitlin, D., Kitzes, J.: Synthesis of serum albumin, embryo-specific $\alpha$-globulin and conalbumin by the chick yolk sac. Biochim. Biophys. Acta. (Amst.) 147, 334-340 (1967)

Gitlin, D., Perricelli, A., Gitlin, G.M.: Synthesis of $\alpha$-fetoprotein by liver, yolk sac and gastrointestinal tract of the human conceptus. Cancer Res. 32, 979-982 (1972)

Gitlin, D., Perricelli, A., Gitlin, J.D.: The presence of serum $\alpha$-fetoprotein in sharks and its synthesis by fetal gastrointestinal tract and liver. Comp. Biochem. Physiol. 46B, 207-215 (1973)

Linder, E., Lahti, A., Saxen, L.: Immunofluorescent localisation of a fetal protein in the mouse yolk sac. Int. Arch. Allergy 40, 411-418 (1971)

Linder, E., Seppala, M.: Localisation of $\alpha$-fetoprotein in the human foetus and placenta. Acta path. microbiol. scand. 73, 565-571 (1968)

Lindgren, J., Vaheri, A., Ruoslahti, E.: Identification and isolation of a fetoprotein in the chicken. Differentiation 2, 233-236 (1975)

Murgita, R.A., Tomasi, T.B.: Suppression of the immune response by $\alpha$-fetoprotein. I. The effect of mouse $\alpha$-fetoprotein on the primary and secondary antibody response. J. exp. Med. 141, 269-286 (1975 a)

Murgita, R.A., Tomasi, T.B.: Suppression of the immune response by $\alpha$-fetoprotein. II. The effect of mouse $\alpha$-fetoprotein on mixed lymphocyte reactivity and mitogen-induced lymphocyte transformation. J. exp. Med. 141, 440-452 (1975b)

Pagé, M.: $\alpha$-fetoprotein: Purification on sepharose-linked concanavalin A. Canad. J. Biochem. 51, 1213-1215 (1973)

Ruoslahti, E., Pihko, H.: Effect of chemical modification on the immunogenicity of homologous $\alpha$-fetoprotein. Ann. N.Y. Acad. Sci. 259, 85-94 (1975)

Sainte-Marie, G.: A paraffin embedding technique for studies employing immunofluorescence. J. Histochem. Cytochem. 10, 250-256 (1962)

Scheidegger, J.J.: Une micro-méthode de l'immunoélectrophorèse. Int. Arch. Allergy. 7, 103-110 (1955)

Slade, B., Budd, S.: Localisation of $\alpha$-fetoprotein in fetal and newborn rabbits. Biol. Neonate 21, 309-320 (1972)

Swartz, S.K., Soloff, M.S.: The lack of oestrogen binding by human $\alpha$-fetoprotein. J. clin. Endocr. 39, 603-606 (1974)

Tartarinov, Y.S., Afanasyeva, A.V.: The detection of similar antigenic determinants in embryospecific alpha-globulin of man and certain animals. Byull. Eksp. Biol. Med. 59, 65-69 (1965)

Uriel, J., Nechaud, B. de, Dupiers, M.: Estrogen-binding properties of rat, mouse and man fetospecific serum proteins. Demonstration by immuno-autoradiographic methods. Biochem. biophys. Res. Commun. 46, 1175-1180 (1972)

Weller, E.M.: Embryo-specific serum proteins in the chick. Tex. Rep. Biol. Med. 24, 164-172 (1966)

Weller, E.M.: Characterisation of embryonic antigens in the plasma of developing chick embryos. Cancer Res. 36, 3415-3422 (1976) 